Original Article

\title{
Diode laser- an ideal tool for inferior turbinate reduction in allergic rhinitis
}

\author{
Satheesh Kumar Bhandary B. ${ }^{1}$, Rishi Ajay Khanna ${ }^{2}$, Vadisha Srinivas Bhat ${ }^{3}$ \& Rajeshwary Aroor ${ }^{4}$ \\ ${ }^{1,3,4}$ Professor, ${ }^{2}$ Senior Resident, Department of Otorhinolaryngology, K.S. Hegde Medical Academy, Nitte University, \\ Mangalore -575018. \\ *Corresponding Author : Satheesh Kumar Bhandary, Professor, Department of Otorhinolaryngology, K S Hegde M edical Academy, \\ Mangalore-575018, Karnataka, India. Mobile: +91984130517, E-mail : sakubaraj@gmail.com.
}

Received

: 12-03-2016

Review Completed : 20-06-2016

Accepted

28-06-2016

Keywords : Allergic rhinitis; inferior turbinate hypertrophy; diode laser; conventional turbinectomy.

\begin{tabular}{|c|}
\hline Access this article online \\
\hline Quick Response Code \\
\hline
\end{tabular}

\begin{abstract}
:
Background: Inferior turbinate hypertrophy is the leading cause of nasal obstruction in subjects with allergic rhinitis. Patients not responding to medical line of treatment can benefit from turbinate reduction surgery, which aims at relieving symptoms.
\end{abstract}

Objective: Our study aimed at comparing the improvement in nasal symptoms as well as complicationsfollowing diode laser turbinoplasty and conventional inferior turbinectomy.

Methods: A comparative interventional study conducted on randomly selected 93 patients of allergic rhinitis with hypertrophied inferior turbinate, attending the department of Otorhinolaryngology, in K.S.Hegde Charitable Hospital, M angalore over a period of 3 years. Fifty patients underwent conventional inferior turbinectomy and 43 underwent diode laser inferior turbinoplasty. All patients were evaluated for improvement in symptoms at follow up on 1 week, 1 month, 3 month and 6 month.

Results: The overall subjective improvement at 6 month was $71.10 \%$ in Conventional turbinectomy group and $73.84 \%$ in Laser turbinoplasty group. Both procedures are equally effective in reducing the turbinate size; however crusting, post-operative pain, blood loss and number of days of nasal pack was found to be significantly less in Laser turbinoplasty group.

Conclusion: Diode laser turbinoplasty and conventional turbinectomy for inferior turbinate hypertrophy are equally good in providing long-lasting symptom reduction in patients of allergic rhinitis with hypertrophied inferior turbinate. However diode laser turbinoplasty is a safe and effective procedure resulting in a controlled coagulation and ablation of the soft tissue with less immediate post-operative pain, bleeding, crusting, duration of nasal pack and hospital stay. The better results of diode laser turbinoplasty provide an excellent patient acceptance.

\section{Introduction}

Allergic rhinitis is a global health problem. Prevalence rate is $15 \%$ in men and $14 \%$ in women. Allergic rhinitis is associated with asthma, otitis media, Eustachian tube dysfunction, sinusitis, nasal polyps, allergic conjunctivitis, atopic dermatitis, and fatigue ${ }^{2}$. In perennial allergic rhinitis, dilatation of venous sinuses in inferior turbinate mucosa causes inferior turbinate hypertrophy, resulting in chronic nasal obstruction ${ }^{3}$.

Various medical therapies, including intranasal spray and anti-allergic medication are often ineffective ${ }^{4}$. Surgical procedures like partial or total turbinectomy, submucosal diathermy and cryotherapy ${ }^{5}$ have proved effective in relieving nasal obstruction in such patients. However, these procedures frequently cause bleeding, pain, oedema and require nasal packing. Recently, the radiofrequency energy powered instrumentation and various kinds of lasers like CO2, KTP, Nd:YAG, Ho:YAG and diode lasers are used for turbinate reduction ${ }^{6}$.

The diode laser $(940 \mathrm{~nm})$ light is absorbed by water and blood thus providing excellent coagulation. This result in reduction of nasal mucosa and improve nasal breathing. Moreover, the diode laser can be used in contact application, offering vaporization with precise tissue cutting ${ }^{7}$. 
Our study aimed at comparing the improvement in symptoms as well as complications following diode laser inferior turbinoplasty and conventional inferior turbinectomy in patients of allergic rhinitis with inferior turbinate hypertrophy.

\section{Materials and methodology}

This is a comparative interventional study, conducted on 93 patients of allergic rhinitis with hypertrophic inferior turbinate, in the department of Otorhinolaryngology, in K. S. Hegde Charitable Hospital, $M$ angalore in the period from July 2010 to June 2013. Ethical clearance was obtained from the institutional ethics committee. Written informed consent was obtained from patients, before enrolling them for the study. Fifty patients underwent conventional inferior turbinectomy and 43 underwent diode laser inferior turbinoplasty. Patients with gross deviated nasal septum, chronic sinusitis, chronic granulomatous disease and sinonasal polyposis were excluded from the study.

The severity of nasal obstruction, sneezing, and rhinorrhoea were recorded and graded based on a 4-point scale: 0 (no symptoms), 1 (mild symptoms), 2 (moderate symptoms), 3 (severe symptoms). Graduated cold spatula test provided an objective evaluation of nasal obstruction. The size of turbinate assessed and graded according to Yanez and Mora $^{8}$ grading; width measured as: 1 (fully retracted), 2 (engorged halfway to nasal fossa), 3 (engorged and reaching nasal septum) and length measured as: 1 (the inferior turbinate not crossing the choanal arch posteriorly), 2 (reaching but not crossing choanal arch line), 3 crossing the choanal line posteriorly.

Diode laser inferior turbinoplasty was done under general anaesthesia. Pre-operatively, topical anaesthetic agent applied for 10 minutes, with 1:200000 adrenaline in 4\% lignocaine. The laser light applied in "contact" mode by using a soft-bending silica fibre (400-mm core diameter) at 10-14 Watt power in the continuous-wave mode. Three to four laser light applications performed by drawing the fibre from the posterior to the anterior part of the inferior turbinates and some additional laser spots onto the head of the turbinate.
For conventional turbinectomy, after infiltrating with 1:200000 adrenaline in 2\% lignocaine, turbinate was medialized using Freer's elevator, crushed with long artery and cut with turbinectomy scissors. The duration of surgery and blood loss during surgery were recorded. Postoperatively, the nasal cavities were packed with sponge in finger glove smeared with antibiotic ointment (Figure 1).

Pack was removed on postoperative day 1 or 2 . All patients were given an antibiotic and analgesic. Post-operative pain was assessed by visual analogscale (VAS).

Changes in nasal symptoms, frequency of asthma attacks and overall quality of life were recorded pre operatively and post operatively at 1 week, 1 month, 3 month and 6 month.

Nasal crusts were classified according to J oniauet al $^{9}(0=$ no crusts; $1=$ a few isolated crusts; $2=$ less than $50 \%$ of the inferior turbinate is crusted; $3=$ more than $50 \%$ of the inferior turbinate is crusted).

\section{Results}

Ninety three patients of allergic rhinitis with hypertrophied inferior turbinate were included in the study. Mean duration of symptoms was 3.63 years in conventional turbinectomy group and 4.59 years in Laser turbinoplasty group.

In conventional turbinectomy group all patients presented with nasal obstruction. After 3 months only 1 patient (2\%) and at 6 months no patient had nasal obstruction. In Laser turbinoplasty group, all patients had nasal obstruction. After 3 months, only 2(4.7\%) patients and at 6 month, no patient had nasal obstruction (Figure 2).

In conventional group 8 (16\%) patients presented with excess nasal secretion. At 3 months, only 1 (2\%) patient and at 6 months no patient had excess nasal secretion. In Laser group 10 (23.3\%) patients had nasal secretion, at 3 months none of the patients had nasal secretion. On comparing the two groups, secretions were reduced earlier (at 3 month) in all patients of Laser group, while at 6 month 
in Conventional group.

In conventional group, 25 (50\%) patients \& in Laser group $26(60.5 \%)$ patients presented with excess sneezing. On comparing the two groups, at 6 month, no sneezing was present in all patients of conventional group, while sneezing persisted in Laser group.

There was no statistically significant difference observed in both groups, for nasal obstruction, secretion and sneezing

On comparing the two groups, no patient had postoperative nasal bleeding after 1 month in Laser group, although it persisted till 3 months in conventional group. But there was no statistically significant difference between two groups.

History of asthma was present in $4(8 \%)$ patients in Conventional group and 4 (9.3\%) patients in Laser group. Six months after surgery, frequency of asthma attacks decreased in $3(75 \%)$ patients of conventional group and in all $4(100 \%)$ patients of Laser group. Asthma attacks persisted in 1 (25\%) patient of Conventional group. No statistically significant difference was observed between two groups. However, asthma attacks were decreased in both the groups, but we could not conclude due to less number of asthmatic patients.

In Conventional group, mean value of cold spatula test (in $\mathrm{cm}^{2}$ of misting) observed on right side was $1.32 \mathrm{~cm}^{2}$ preoperatively, $1.65 \mathrm{~cm}^{2}$ at 3 month and $1.93 \mathrm{~cm}^{2}$ at 6 month. On left side, the values were $1.29 \mathrm{~cm}^{2}$ pre-operatively, 1.69 $\mathrm{cm}^{2}$ at 3 month and $1.86 \mathrm{~cm}^{2}$ at 6 month.

In Laser group, mean value of cold spatula test observed on right side was $1.12 \mathrm{~cm}^{2}$ pre-operatively, $1.60 \mathrm{~cm}^{2}$ at 3 month and $1.77 \mathrm{~cm}^{2}$ at 6 month and on left side it was as $1.12 \mathrm{~cm}^{2}$ pre-operatively, $1.81 \mathrm{~cm}^{2}$ at 3 month and $1.98 \mathrm{~cm}^{2}$ at 6 month.

On comparing both the groups at 6month, there was significant improvement in nasal airway in both the groups and no statistically significant difference between the groups.
In Conventional group, the grade of right turbinate hypertrophy observed pre operatively was: grade-4 in 1 $(2 \%)$, grade-3 in 39 (78\%), grade- 2 in 9 (18\%), grade- 1 in 1 (2\%) patient. At post-operative 6 month, it was grade-1 in all $50(100 \%)$ patients. The grade of left turbinate hypertrophy observed pre operatively was: grade-3 in 24 (48\%), grade- 2 in 24 (48\%), grade- 1 in 2 (4\%) patients, and at post-operative 6 month was grade- 2 in 9 (18\%), grade-1 in $41(82 \%)$ patients.

In Conventional group, most of patients who presented with grade 3 hypertrophic turbinate pre-operatively were reduced to grade 1 at 6 month.

In Laser group, the grade of right turbinate hypertrophy observed pre operatively was: grade- 4 in $2(4.7 \%)$, grade-3 in $32(74.4 \%)$, grade-2 in $6(14 \%)$, grade-1 in $3(6.9 \%)$ patients. At post-operative 6 months, it was grade- 2 in 5 (11.6\%), grade-1 in $38(88.4 \%)$ patients. The grade of left turbinate hypertrophy observed pre operatively was: grade-3 in $3(7 \%)$, grade- 2 in 36 (83.7\%), grade- 1 in $4(9.3 \%)$ patients. At post-operative 6 months, it was grade- 2 in 4 (9.3\%), grade-1 in 39 (90.7\%) patients.

In Laser group most of patients presented with grade 3 hypertrophic turbinate pre-operatively, which was reduced to grade 2 and grade 1 in most of patients at 6 months.

However on comparing the two groups at 6 month there was significant decrease in turbinate size in conventional group with very high statistically significant difference for right turbinate size at 6 month $(p=0.019)$.

In conventional group, crusting observed at 1 month as: grade- 3 in $1(2 \%)$, grade- 2 in $32(64 \%)$, grade- 1 in $15(30 \%)$, grade- 0 in 2 (4\%) patients. Crusting at 3 month was: grade2 in $1(2 \%)$, grade- 1 in 8 (16\%), grade-0 in 41 (82\%) patients. No patient had crusting at 6 month.

In Laser group, crusting observed at 1 month as: grade-1 in 21 (48.8\%), grade-0 in 22 (51.2\%) patients. Crusting at 3 month was: grade-1 in 2 (4.7\%), grade-0 in 41 (95.3\%) patients. No patient had crusting at 6 month. 
However on comparing the two groups at 1 month, many patients of Laser group had grade-1 crusts, while conventional group had grade-2 crusts. Crusting was less in Laser group (with very high statistically significant difference at 1 month for crusting, $p<0.001$ ).

At 1 week, synechiae was observed in $6(12 \%)$ patients in conventional group and $2(4.7 \%)$ patients in Laser group. At 1 month 2 (4\%) patient in conventional group and 1 (2.3\%) patient Laser group had synechiae and synechiae release was done in outpatient department. No patient had synechiae after 3 months in either group. On comparing the two groups, synechiae were seen more in conventional groups but no statistically significant difference observed.

Intra-operative mean blood loss was $22.56 \mathrm{ml}$ in conventional group and $13.39 \mathrm{ml}$ in Laser group, which was more for conventional group. Average operative time taken for the procedure was 8.94 min for conventional group and 21.67 min for Laser group, which more for Laser group.

Mean number of days nasal pack kept were 1.98 days in conventional group and 1 day in Laser group; with more duration of nasal pack requirement in conventional group. Mean duration of hospital stay was 4.1 days for conventional group and 3.0 days for Laser group; with more duration of hospital stay in conventional group, which was due to the delay in nasal pack removal. Duration of nasal pack, hospital stay, blood loss and operative time had very high significant statistical difference, $p<0.001 \mathrm{VHS}$.

Postoperative pain scores (mean value) on post-operative day-1 was 4.72 in conventional group and 3.86 in Laser group, on day-2, the score was 3.74 in conventional group and 1.05 in Laser group, with more pain score in conventional group, with very high significant statistical difference, $p<0.001 \mathrm{VHS}$. In both the methods, no other complications were encountered.

\section{Discussion}

Surgical reduction of the hypertrophied inferior turbinate is the treatment of choice in patients with allergic rhinitis, when nasal obstruction is unresponsive to medication ${ }^{10}$. Techniques which destroy the turbinate mucosa lead to
Figure 1 : Sponge in finger glove pack used for anterior nasal packing after turbinate reduction surgery

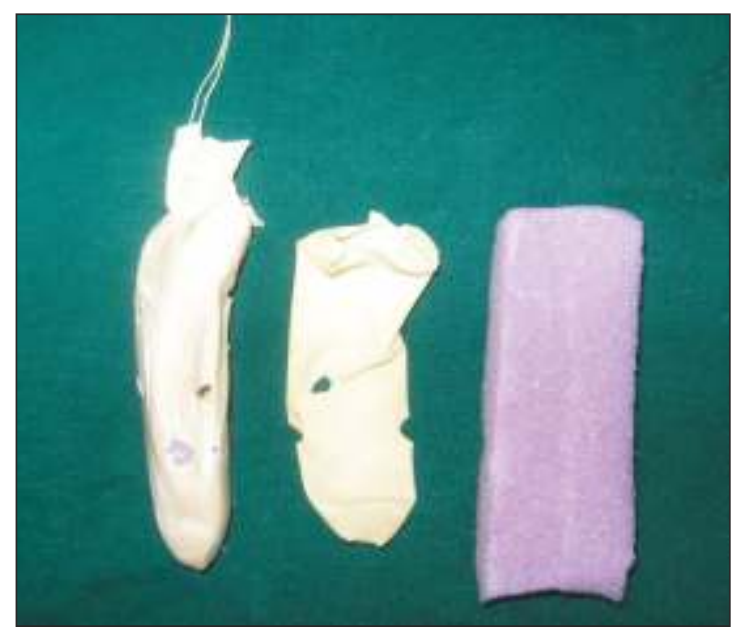

Figure 2: Comparison of nasal obstruction in conventional and diode laser turbinoplasty groups

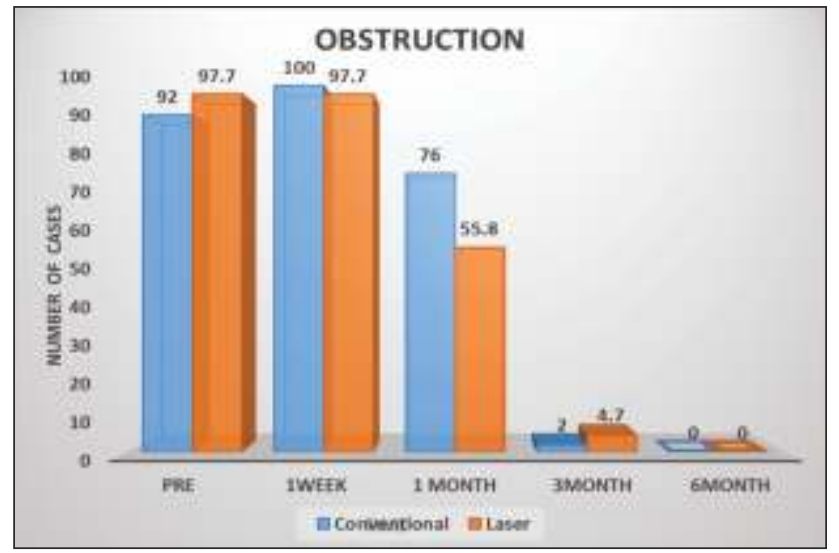

loss of turbinate function along with crusting and adhesions $^{11}$. On the other hand, laser turbinoplasty, which preserves turbinate function and nasal mucosa for mucociliary clearance, also has some limited short-term disadvantages ${ }^{12}$.

Laser turbinoplasty has the advantages of mucosal preservation, controlled volume reduction, minimal trauma, enhanced precision, reduced bleeding, lower post-operative pain and crusting with shorter duration of nasal pack, hospital stay and providing marked improvements in nasal obstruction ${ }^{13}$.

In our study, on 1 week after surgery, nasal obstruction was present in most of the patients in both the groups, due to postoperative mucosal edema, blood clots and crusting. However at 6 month, no statistically significant difference 
was seen between both the groups. This signifies that, both the procedures are equally good in reducing nasal obstruction.

This observation was consistent with study of Rahman M Z et $\mathrm{al}^{14}$. In their study 24(39.34\%) patients underwent conventional turbinectomy and $37(60.65 \%)$ patients underwent diode laser turbinoplasty. They found that $32(86.48 \%)$ patients in Laser group and 20(79.16\%) patients in non-laser group were symptoms free at 6 month. In our study, nasal secretion is reduced in both groups, with more significant reduction seen in Laser group. This is probably due to local effect of laser energy causing controlled coagulation and ablation of the adjacent glands and nasal mucosa ${ }^{1}$.

This finding was consistent with study of Hoque M R et al'. In their study 45 patients underwent diode laser turbinoplasty. Nasal secretion was present in 22 (49\%) patients pre-operatively. After 6 months secretion was reduced in $13(60 \%)$, no change in $6(27 \%)$, worsening in $2(9 \%)$ and new appearance in $1(4 \%)$ patient. In our study at 6 month no patient in both the groups had secretion.

Postoperative nasal bleeding was seen more in patients of conventional group. Two patients were readmitted and endoscopic cauterization was done. Our findings are consistent with the study of Rahman M Zet al ${ }^{14}$.

Hoque MR et $\mathrm{al}^{7}$ reported that the mean operation time was $8 \mathrm{~min} /$ turbinate, no nasal packing was necessary and no immediate complications were observed.

In our study, allergic rhinitis was associated with asthma, in $4(8 \%)$ patients of Conventional group and in $4(9.3 \%)$ patients of Laser group. Post operatively after 6 months frequency of asthma attacks decreased in both the groups.

This finding is consistent with the study of Ducroz Vet al. ${ }^{15}$ They studied Inferior turbinectomy in asthmatic children. Nineteen children with asthma underwent bilateral inferior turbinectomy. Mean age was 11.5 years (range 817). They reported, a clear improvement in 15 (79\%), partial improvement in $3(16 \%)$ and unilateral improvement in 1 (5\%) patient. They concluded that, inferior turbinectomy provides considerable improvement in the comfort of patients with asthma, especially in terms of nasal ventilation. But due to small sample size of asthmatic patients, we could not come to conclusion about this observation. By both the procedure, reduction in turbinate size achieved at 6 month was equal without any statistically significant difference.

Objective assessment of nasal airway by cold spatula test revealed, decreased misting at 1 week after the surgery due to crusting and edema, which is followed by an improvement in nasal airway after 3 and 6 months in both groups. However, there was no statistically significant difference between two groups.

These findings were consistent with. Caffier PP et a ${ }^{16}$. They studied therapeutic effect of diode laser inferior turbinate reduction on nasal obstruction and decongestant abuse in perennial and seasonal allergic rhinitis on 40 patients (20 perennial, 20 seasonal).The improvement was greatest for nasal obstruction, initially higher in perennial allergic rhinitis but more sustained in seasonal allergic rhinitis.

In our study the intraoperative blood loss during laser turbinoplasty was significantly less than conventional turbinectomy with $p<0.001$, which is very highly significant.

Crusting observed was more in conventional group than the Laser group. There was significant less amount of crusting in Laser group, which is with $p<0.001$, which is very highly statistically significant.

This finding was consistent with study of Rahman M Zet al ${ }^{14}$. In their study 24 (39.34\%) patients underwent conventional turbinectomy and $37(60.65 \%)$ patients underwent diode laser turbinoplasty. Only 8 patients (21.62\%) presented with crusting in Laser group as compared to 22 patients (91.66\%) in non-laser group.

Caffieret PP al ${ }^{16}$, also observed that, postoperative edema disappeared within the first week and crusting within 6 weeks after surgery in all patients of diode laser turbinoplasty. 
The mean operative times for conventional turbinectomy was significantly less as compared to laser turbinoplasty with $p<0.001$, which is very highly significant.

We found significantly low intra-operative blood loss and decreased hospital stay in Laser group, as compare to conventional group. Duration of nasal pack kept was also shorter in Laser group with $p<0.001$, which is very highly significant.

Post-operative pain was also significantly less in Laser. This is consistent with Caffier PP et a $\mathrm{al}^{16}$. They mentioned that, VAS (visual analog scale) was characterized by very low values for intraoperative pain and discomfort and high postoperative patient satisfaction.

The present study signifies the usefulness of the diode laser for intranasal surgery. In contact mode, it provides an excellent coagulation of soft tissue, a controllable performance, and a good hemostasis in a mean operation time of 8 minutes. With respect to $\mathrm{CO}_{2}, \mathrm{Nd}$ :YAG, $\mathrm{KTP}$, Argon-

\section{References}

1. Togias AG. Systemic immunologic and inflammatory aspects of allergic rhinitis. J Allergy ClinImmunol. Nov 2000;106(5 Suppl):S247-50.

2. Druce HM . Allergic and nonallergic rhinitis. In: M iddleton EM Jr, Reed $\mathrm{CE}$, Ellis EF, Adkinson NF Jr, Yunginger JW, Busse WW, eds. Allergy: Principles and Practice. $5^{\text {th }}$ ed. St. Louis, Mo: Mosby Year-Book; 1998:1005-16

3. CraigTJ, Teets S, Lehman EB, Chinchilli VM , Zwillich C. Nasal congestion secondary to allergic rhinitis as a cause of sleep disturbance and daytime fatigue and the response to topical nasal corticosteroids. J Allergy Clin Immunol. M ay 1998;101(5):633-7.

4. Mucci S, Sismanis A. Inferior partial turbinectomy: an effective procedure for chronic rhinitis. ENTJ 1994;73:405-7.

5. Ophir D, Shapira A, Marshak G. Total inferior turbinectomy for nasal airway obstruction. Arch Otolaryngol Head Neck Surg 1985;111(2):935.

6. Min YG, Kim HS, Yun YS, Kim CS, Jang YJ, Jung TG. Contact laser turbinate surgery for the treatment of idiopathic rhinitis. Clin Otolaryngol Allied Sci. 1996;21(6):533-6.

7. Hoque M R, Rasel A, Asad MK, Aich M L. Turbinoplasty of hypertrophied inferioriturbinate by diode laser. Bangladesh J Otorhinolaryngol 2010; ion, and Ho:YAG lasers, the advantages of the diode laser in endonasal surgery are good coagulation properties, low costs, and the small size of the device. High patient acceptance, less bleeding, less duration of hospital stay hospitalization favors, this therapeutic modality.

\section{Conclusion}

The results of our study confirmed that, in patients of allergic rhinitis with inferior turbinate hypertrophy, the diode laser turbinoplasty and conventional turbinectomy are equally effective in providing long-lasting symptom reduction. But diode laser turbinoplasty is a safe and effective procedure, resulting in a controlled coagulation and ablation of the soft tissue with less immediate postoperative pain, bleeding, crusting, duration of nasal packing and duration of hospital stay. Diode laser turbinoplasty offers more precision, gentleness, control and less postoperative discomfort. The better results of diode laser turbinoplasty provide an excellent patient acceptance.

16(1): 29-34

8. Yanez C, Mora N: Inferior turbinate debriding technique: ten-year results. Otolaryngol Head Neck Surg 2008;138:170-175.

9. Joniau S, Wong I, Rajapaksa S, et al: Long-term comparison between submucosal cauterization and powered reduction of the inferior turbinates. Laryngoscope 2006;116:1612-1616.

10. Lee CF, Chen TA: Power microdebrider- assisted modification of endoscopic inferior turbinoplasty: a preliminary report. Chang Gung MedJ (English edition) 2004; 27(5): 359-365.

11. Sapci T, Sahin B, Karavus A, et al: Comparison of the effects of radiofrequency tissue ablation, $\mathrm{CO}_{2}$ laser ablation, and partial turbinectomy applications on nasal mucociliary functions. Laryngoscope 2003; 113: 514-519.

12. Hol M, Huizing E: Treatment of inferior turbinate pathology: a review and critical evaluation of the different techniques. Rhinology 2000; 38 : 157-166.

13. Eichler J, Goncalves, O. A review of different lasers in endonasal surgery: Ar-, KTP-, Dye-, Diode-, Nd-, Ho-, and CO(2)-laser. Medical Laser Application 2002; 